\title{
The role of thought suppression in conversion disorder in relation to depression, symptom interpretation and sleep hygiene: a case-control study
}

\author{
PINAR GÜZEL ÖZDDEMIR ${ }^{1}$ \\ https://orcid.org/0000-0002-2135-2553 \\ UMUT KIRLI1,2 \\ https://orcid.org/0000-0001-9924-2672 \\ Mesut IșiK ${ }^{1}$ \\ https://orcid.org/0000-0003-1707-7402 \\ ŞÜHEDA TAPAN ${ }^{1}$ \\ https://orcid.org/0000-0002-3809-4685 \\ 1 Department of Psychiatry, Faculty of Medicine, Yuzuncu Yil University, Van, Turkey. \\ 2 Department of Psychiatry and Neuropsychology, School for Mental Health and Neuroscience (MHeNS), European Graduate School of Neuroscience (EURON), Maastricht University Medical \\ Centre, Maastricht, The Netherlands.
}

Received: 03/24/2019 - Accepted: 11/04/2019

DOl: 10.1590/0101-60830000000233

\begin{abstract}
Background: Thought suppression has been associated with a number of psychiatric disorders. However, the association with conversion disorder (CD) has not been investigated yet. Objective: To investigate the role of thought suppression in CD. Methods: Eighty consecutive outpatients with a diagnosis of CD and sixty age, sex and neighborhood-similar controls were evaluated using Beck Depression Inventory-I (BDI-I), White Bear Suppression Inventory (WBSI), Symptom Interpretation Questionnaire (SIQ) and Sleep Hygiene Index (SHI). Cases and controls were compared in regard to thought suppression scores considering their status of high depression scores via a logistic regression model. The reciprocal associations of thought suppression with other clinical dimensions in $\mathrm{CD}$ were assessed. Finally, structural equation modelling was applied to untangle the possible connections. Results: CD patients had significantly higher scores of thought suppression than the control group. However, the difference was below the significance level when CD patients without comorbid high depression scores were taken into account. Thought suppression was associated with the clinical severity of CD. According to the structural equation model, older age and somatic attributions to the common bodily sensations were the significant correlates of thought suppression among CD patients. Discussion: Thought suppression may be considered as a non-specific marker of clinical severity in CD.
\end{abstract}

Özdemir PG et al. / Arch Clin Psychiatry. 2020;47(3):59-64

Keywords: Conversion, thought suppression, depression, sleep hygiene, somatic attribution.

\section{Introduction}

Conversion disorder (CD) - also addressed as functional neurological symptom disorder - manifests with neurological symptoms incompatible with the recognized conditions. To date, some models on the onset and course of these symptoms have been proposed ${ }^{1}$. One of these models suggests that symptoms are indeed blurry observed dissociated materials which partly intrude into awareness in response to a reminder ${ }^{2}$. Another model conceptualizes symptoms as basic biological responses aiming to regulate arousal against acute threats or post-traumatic reactions ${ }^{3}$. An alternative model proposes that symptoms occur in the context of operant conditioning and/or are driven by primary or secondary gains ${ }^{4}$. Finally, these symptoms are considered as a part of a defensive mechanism against emotional awareness. An important proportion of these models refer to thought suppression which is a type of motivated forgetting process aiming to alleviate conflicting thoughts and feelings ${ }^{5}$. Accordingly, it may be proposed that stress-related thoughts and images are partly replaced by bodily symptoms and related thoughts at the conscious level ${ }^{6}$. Recent studies provided some indirect evidence for the association between CD and thought suppression. CD patients were found to have elevated rates of both severe life events history and "escape" from stressors7. Furthermore, a functional magnetic resonance imaging (fMRI) study demonstrated abnormal activities in brain areas related to memory and emotion during the recall of the etiologically relevant event in $\mathrm{CD}$ patients 8 . Surprisingly, no studies to date have directly investigated the role of thought suppression in CD.

In addition to the potential association with $\mathrm{CD}$, thought suppression was associated with tendency to somatization ${ }^{5}$. On the other hand, many studies consistently showed higher somatization sub-scale scores in CD mainly independent of the levels of anxiety, depression or trauma-exposure. Relatedly, psychosomatic symptom "reporting" level was associated with the clinical severity of CD". Taken together, these clues suggest potential interactions between thought suppression and tendency to somatization in CD.

Thought suppression was associated with poor sleep hygiene via exacerbation of intrusive thoughts at sleep onset and increased occurrence of the suppressed thoughts in dreams. At the same time, thought suppression was associated with depression via a similar mechanism ${ }^{5}$. Furthermore, poor sleep hygiene was shown to be common and associated with functional impairment in $\mathrm{CD}^{10}$. Similarly, depression is one of the most prevalent conditions cooccurring with $\mathrm{CD}$. Finally, poor sleep quality is one of the core features of depression. From this point of view, evaluation of the complex interactions between thought suppression, sleep hygiene, depression and tendency to somatization in CD may provide important insights into the underlying mechanisms of the disorder. However, no studies to date have investigated these interactions in CD.

The aims of this paper were to analyze:

(i) The difference in thought suppression scores between CD patients and individuals with no current or past functional neurological symptom;

(ii) The specificity of thought suppression to CD;

(iii) The complex associations between thought suppression and other clinical entities including sleep hygiene, depression, reported number of common bodily sensations and attributions to these sensations in CD. 
It is hypothesized that CD patients will have more thought suppression scores than the control group. Furthermore, thought suppression scores will be higher in CD patients without comorbid high depression scores. Finally, thought suppression will be associated with more common bodily sensations, more severe depressive symptoms and worse sleep hygiene among CD patients.

\section{Methods}

\section{Participants}

A hundred consecutive patients who attended to the outpatient clinic and diagnosed with CD between June 2015 and May 2016 were referred to this case-control study. In order to make differential diagnosis with CD and assess the comorbid and past diagnoses of the participants, clinical psychiatric interviews were conducted using DSM-5 criteria by two separate psychiatrists (junior psychiatrist: in charge of the outpatient clinic; senior psychiatrist: performing the clinical interviews for this study). The inclusion criteria for the study were as follows: Being 18 to 50 years of age with a referral from the outpatient clinic due to a primary diagnosis of $C D$ and volunteering to participate in the study. The exclusion criteria was having a diagnosis of a schizophrenia spectrum and other psychotic disorders, disorders with psychotic features specifier, substance-related and addictive disorders, neurodevelopmental disorders, neurocognitive disorders or progressive neurological diseases. The upper limit of the age range was set to 50, because of the increasing incidence of minor cognitive disabilities above this age. Furthermore, individuals with the above-mentioned comorbid psychiatric diagnoses were excluded because of the potential cognitive deficits associated with these disorders. Neurological examinations were performed to all cases by the consulting neurologist in order to exclude the possibility of a neurological cause. Twenty patients who had been referred to the study were excluded by the senior psychiatrist due to the DSM-5 Criterion C of CD: "The symptom or deficit is not better explained by another medical or mental disorder". In more detail, the primary diagnoses changed to panic disorder in twelve patients, to somatic symptom disorder in four patients, to post-traumatic stress disorder (PTSD) in three patients, to depersonalization/ derealization disorder in one patient in the second clinical interview. No cases were excluded due to the exclusion criteria of the comorbid diagnosis. Consequently, eighty cases were included in the study. Control group was selected via local announcements to the nearest household to the case's address in order to control for the confounders related to sub-cultural aspects. Junior psychiatrist invited eighty age (within five years) and sex-similar volunteers with no current or past symptoms of altered voluntary motor or sensory function and no self-reported disorders within the exclusion criteria. Sixty-six individuals attended to the research hospital in response to these invitations. Six individuals were excluded as a result of the clinical interview performed by the senior investigator (due to the history of the disorders within the exclusion criteria). Consequently, control group included sixty individuals.

\section{Ethical standards}

This study was conducted according to tenets of the Declaration of Helsinki and approved by the Clinical Ethics and Research Committee of Yuzuncu Yil University, Faculty of Medicine. All participants signed an informed consent form declaring that they had been fully informed of the purposes and conduct of the study. Participants were not paid for their participation.

\section{Assessments}

After the clinical interview by the senior psychiatrist for the inclusion and exclusion criteria, background information concerning selfreported somatic and psychiatric disorder history was collected through an interview by the junior psychiatrist. Then, the scales were administrated to the cases and controls accompanied by the junior psychiatrist. All participants were able to complete scales. No time limit was set to complete the scales. It took about 1 hour in average to complete the scales.

Beck Depression Inventory Version I (BDI-I) was used to assess the severity of depressive symptoms. BDI-I score was evaluated in two ways: i) a total sum score obtained by adding the points for the 21 items, ranging from zero to 63 points ii) a cut-off value of 17 points was used to define the participants with high depression scores, accepted as the reference assessment rate in Turkish population ${ }^{11}$. Mean internal-consistency estimates of the total scores were obtained as 0.86 for psychiatric patients. The mean correlation between the BDI total score and clinical ratings of depression was demonstrated to be greater than $0.60^{12}$. The Turkish version of the instrument was used in various surveys, and showed reasonable internal consistency $($ Cronbach's alfa $=0.8)$ and concurrent validity $(r=0.63)^{11}$.

Symptom Interpretation Questionnaire (SIQ) was used to assess different styles of causal attributions about the common bodily sensations. SIQ includes 13 common bodily sensations, evaluates the number of common bodily sensations experienced as well as somatic, psychological and normalizing attributions to these sensations. Respondents indicate how much each attribution is related to each bodily sensation. Psychological, somatic, and normalizing scales were demonstrated to have reasonable internal consistency with alphas of $0.88,0.73$, and 0.79 , respectively ${ }^{13}$. The Turkish version of the questionnaire has two differences from the original version: The four-point Likert type was changed to five-point Likert type and a new item $\left(14^{\text {th }}\right)$ was added. The SIQ Turkish version in its adapted form was used in different samples and showed satisfactory criterion related validity, discriminating power for specific groups and construct validity. Furthermore, psychological, somatic, and normalizing subscales of the Turkish version in its adapted form showed good internal consistency with Cronbach's alpha values of $0.87,0.87$ and 0.86 , respectively ${ }^{14}$.

White Bear Suppression Inventory (WBSI) was used to assess the individual's tendency to suppress unwanted intrusive thoughts ${ }^{15}$. WBSI is a 5-point Likert type self-report inventory consisting of 15 items. The total score ranges from 15 to 75 . Higher WBSI scores reflect greater suppression of unwanted thoughts. To date, the inventory has been used in samples with various disorders including obsessive compulsive disorder, anxiety disorders and depression. Previous studies indicated high internal reliability of WBSI (Cronbach alphas of 0.87-0.89 across several studies) ${ }^{15}$. Turkish version of the instrument was used in different samples, and showed high internal consistency $(\alpha=0.92)$ and appropriate discriminating power ${ }^{16}$

Sleep Hygiene Index (SHI) was used to assess environmental and behavioral variables that could promote inadequate sleep ${ }^{17}$. SHI consists of 13-items derived from the diagnostic criteria for inadequate sleep hygiene in the International Classification of Sleep Disorders (ICSD). Individuals are requested to provide information on the frequency of these behaviors. Item scores are summed up providing a global assessment of sleep hygiene ranging from 13 to 65 . Higher scores are indicative of worse sleep hygiene practices. The original version of the scale showed a superior internal consistency $(\alpha=0.66)$ to previous sleep hygiene instruments and a good test-retest reliability $(\mathrm{r}=0.71)(17)$. The Turkish version of the scale was used in different samples, and showed satisfactory internal consistencies both in community-based and in clinical samples with Cronbach's alpha values of 0.70 and 0.71 as well as adequate concurrent validities ${ }^{18}$.

\section{Statistical analysis}

All analyses were conducted using the software package STATA, version 13 (StataCorp, 2013). First, the CD patients were compared with the control group in terms of demographic variables using the chi-square test and t test where appropriate. Results were presented with effect size measures (Cramer's $V$ for chi-square test and Cohen's $d$ for t test). Then, CD patients and the control group were compared in terms of thought suppression scores using logistic regression. To evaluate the specificity of thought suppression to $\mathrm{CD}$, an independent variable combining $\mathrm{CD}$ and high depression scores was constructed 
(0: no $C D$, no high depression scores; $1: C D$, no high depression scores; 2: no CD, high depression scores; 3 : both CD and high depression scores). This variable was used in a logistic regression model of the outcome variable thought suppression score. Subsequently, the model included age, sex and educational level considering the possible influence on thought suppression scores. Results were presented showing both the unadjusted and the adjusted odds ratios (OR) with the 95\% confidence intervals (CI). Finally, in order to evaluate the complex associations between thought suppression and other clinical entities in CD, a two-step analysis were carried out. First, Pearson correlation coefficients (two-tailed) of the thought suppression, attributions to the common bodily sensations, depression and sleep hygiene scores as well as the number of bodily sensations were computed. Guided by previous literature ${ }^{19}$, Pearson correlations $(r)$ with absolute values $<0.3$ were evaluated as weak, 0.3 to $<0.5$ as moderate and $\geq 0.5$ as strong correlations. Then, regarding the multivariate relations between variables, a structural equation model (SEM) was run to investigate the associations of thought suppression score with scores of depression, sleep hygiene, number of bodily sensations and somatic attributions to the common bodily sensations as well as age and sex. The SEM was specified in such a way that complies with flexible modelling paradigm using modification indexes in each step of the analysis. Model fit was assessed using the chi-square. In all analyses, alpha was set at 0.05 .

\section{Results}

\section{Sample characteristics}

The sample characteristics of the case and control group were presented in Table 1 . The sex distribution, age and proportion of participants reporting general medical condition did not significantly differ between the CD patients and the control group. Participants of the control group had significantly higher educational level and were more non-married in comparison with the CD patients (Table 1).

\section{Thought suppression in presence of conversion disorder and high depression scores}

CD patients had significantly higher scores of thought suppression in comparison with the control group (WBSI score: CD patients $37.2 \pm 10.9$ vs. control group $30.1 \pm 15.3, \mathrm{t}=3.2, \mathrm{p}=0.001) . \mathrm{CD}$ patients had 2.6-fold increase in risk of high depression scores in comparison with the control group (OR: 2.6, 95\% CI: 1.7 to 4.0, $\mathrm{p}<0.001)$. Furthermore, CD patients had higher SHI scores than the control group, indicative of worse sleep hygiene practices (OR: 1.17, 95\% CI: 1.11 to $1.24, \mathrm{p}<0.001)$. In comparison with the reference category of no $C D$, no high depression scores; $C D$ in isolation group was not significantly associated with thought suppression scores. However, high depression scores in isolation group was significantly associated with thought suppression scores. The latter association remained significant when adjusted for age, sex and educational level. Details of the associations between thought suppression scores and $\mathrm{CD}$ stratified by presence of comorbid high depression scores were depicted in Table 2.

\section{Correlates of thought suppression among conversion disorder patients}

Pearson correlations of the WBSI, BDI, SHI and SIQ scores among CD patients were presented in Table 3. Thought suppression score was moderately correlated with depression, psychological and somatic attributions to common bodily sensations scores. Furthermore,

Table 1. Demographic variables of the participants with conversion disorder (CD) and the control group

\begin{tabular}{|c|c|c|c|c|c|c|c|c|}
\hline & & \multicolumn{2}{|c|}{$\begin{array}{c}\text { CD patients } \\
(\mathrm{n}=80)\end{array}$} & \multicolumn{2}{|c|}{$\begin{array}{l}\text { Controls } \\
(\mathrm{n}=60)\end{array}$} & \multirow[b]{2}{*}{$t$} & \multirow[b]{2}{*}{ Cohen's $d$} & \multirow[b]{2}{*}{$\mathrm{p}$} \\
\hline & & Mean & SD & Mean & SD & & & \\
\hline Age (y) & & 27.25 & 9.5 & 24.91 & 6.0 & 1.66 & 0.28 & 0.09 \\
\hline \multirow[t]{2}{*}{ Educational level (y) } & & 8.81 & 4.8 & 15.86 & 0.7 & -11.1 & -1.9 & $<0.001$ \\
\hline & & $\mathrm{N}$ & $\%$ & $\mathrm{~N}$ & $\%$ & $\chi^{2}$ & Cramer's V & $p$ \\
\hline \multirow[t]{2}{*}{ Sex } & Male & 18 & $22.5 \%$ & 21 & $35.0 \%$ & 2.66 & 0.13 & 0.103 \\
\hline & Female & 62 & $77.5 \%$ & 39 & $65.0 \%$ & & & \\
\hline \multirow[t]{2}{*}{ Marital status } & Non-married & 36 & $45.0 \%$ & 47 & $78.3 \%$ & 15.78 & 0.34 & $<0.001$ \\
\hline & Married & 44 & $55.0 \%$ & 13 & $21.7 \%$ & & & \\
\hline \multirow[t]{2}{*}{ General medical condition } & Yes & 13 & $16.2 \%$ & 4 & $6.7 \%$ & 2.95 & 0.14 & 0.086 \\
\hline & No & 67 & $83.8 \%$ & 56 & $93.3 \%$ & & & \\
\hline \multirow[t]{4}{*}{ Symptoms } & Attacks or seizures & 49 & $61.2 \%$ & & & & & \\
\hline & Anaesthesia or sensory loss & 25 & $31.2 \%$ & & & & & \\
\hline & Weakness or paralysis & 3 & $3.8 \%$ & & & & & \\
\hline & Speech symptoms & 3 & $3.8 \%$ & & & & & \\
\hline \multirow[t]{2}{*}{ Life events } & Yes & 38 & $47.5 \%$ & & & & & \\
\hline & No & 42 & $52.5 \%$ & & & & & \\
\hline
\end{tabular}

CD: conversion disorder.

Table 2. Associations between thought suppression scores and conversion disorder stratified by the presence of comorbid depression

\begin{tabular}{|c|c|c|c|c|c|c|}
\hline \multicolumn{2}{|c|}{ Diagnosis } & WBSI Score & \multicolumn{2}{c|}{ Model 1 } & \multicolumn{2}{c|}{ Model 2* } \\
\hline Category & $\mathrm{N}(\%)$ & Mean (SD) & $\mathrm{B}(\mathrm{Cl})$ & $\mathrm{p}$ & $\mathrm{B}^{*}(\mathrm{CI})$ & $\mathrm{p}$ \\
\hline $\mathrm{CD}(-)$ Depression (-) & $43(30.7)$ & $26.9(15.3)$ & ref & ref & ref & ref \\
\hline $\mathrm{CD}(+)$ Depression (-) & $20(14.3)$ & $33.5(10.2)$ & $0.57(-0.33-1.49)$ & 0.215 & $0.78(-0.41-1.96)$ & 0.201 \\
\hline $\mathrm{CD}(-)$ Depression (+) & $17(12.1)$ & $38.2(12.2)$ & $1.47(0.45-2.51)$ & 0.005 & $1.63(0.55-2.72)$ & 0.003 \\
\hline $\mathrm{CD}(+)$ Depression $(+)$ & $60(42.9)$ & $38.5(11.0)$ & $1.54(0.81-2.27)$ & $<0.001$ & $1.71(0.78-2.64)$ & $<0.001$ \\
\hline
\end{tabular}

\footnotetext{
*Adjusted for age, sex and educational level. CD: conversion disorder.
} 
Table 3. Pearson correlations between the scales in patients with conversion disorder

\begin{tabular}{|c|c|c|c|c|c|c|c|}
\hline & $\mathrm{BDI}$ & WBSI & SHI & $\begin{array}{l}\text { SIQ Psychological } \\
\text { attribution }\end{array}$ & $\begin{array}{c}\text { SIQ Somatic } \\
\text { attribution }\end{array}$ & $\begin{array}{l}\text { SIQ Normalizing } \\
\text { attribution }\end{array}$ & $\begin{array}{l}\text { SIQ Number of } \\
\text { symptoms }\end{array}$ \\
\hline $\mathrm{BDI}$ & 1 & & & & & & \\
\hline WBSI & $0.33^{*}$ & 1 & & & & & \\
\hline SHI & $0.42^{* *}$ & 0.1624 & 1 & & & & \\
\hline $\begin{array}{l}\text { SIO Psychological } \\
\text { Attribution }\end{array}$ & $0.5987^{* *}$ & $0.363^{* *}$ & $0.3063^{* *}$ & 1 & & & \\
\hline $\begin{array}{l}\text { SIO Somatic } \\
\text { Attribution }\end{array}$ & $0.2302^{*}$ & $0.3024^{* *}$ & 0.1547 & $0.4449^{* *}$ & 1 & & \\
\hline $\begin{array}{l}\text { SI0 Normalizing } \\
\text { Attribution }\end{array}$ & $0.2247^{*}$ & $0.2838^{* *}$ & 0.2233 & $0.4634^{* *}$ & $0.7329^{* *}$ & 1 & \\
\hline $\begin{array}{l}\text { SIO Number of } \\
\text { Symptoms }\end{array}$ & $0.3816^{* *}$ & $0.2562^{*}$ & $0.2611^{*}$ & $0.4499^{* *}$ & $0.2661^{*}$ & 0.0692 & 1 \\
\hline
\end{tabular}

BDI: Beck Depression Inventory; WBSI: White Bear Suppression Inventory; SHI: Sleep Hygiene Index; SIQ: Symptom Interpretation Questionnaire. * $p<0.05 .{ }^{* *} p<0.001$.

thought suppression score was weakly correlated with normalizing attribution to common bodily sensations and number of bodily sensations. However, there was not a significant correlation between thought suppression and sleep hygiene scores. Sleep hygiene in CD had a significant negative correlation with severity of depressive symptoms and number of bodily sensations. Furthermore, sleep hygiene in CD had a significant negative correlation with psychological attributions to common bodily sensations but the correlations with somatic or normalizing attributions were below the significance level.

The correlations of reported number of bodily sensations with depression scores and attribution to common bodily sensations styles were assessed as a sensitivity analysis. As expected, number of bodily sensations was significantly correlated with scores of depression, psychological and somatic attributions but not with normalizing attributions to the common bodily sensations.

The multivariate associations of thought suppression score with other clinical entities in CD as well as age and sex were assessed via a SEM. Model chi-square test revealed adequate goodness of fit $\left(\chi^{2}=2.1, \mathrm{df}=1, \mathrm{p}=0.14\right)$. In the model, as seen in Figure 1, thought suppression score was significantly associated with age and the somatic attribution score $(\mathrm{p}<0.01)$ and was borderline associated with the depression score $(\mathrm{p}=0.06)$. However, no large or significant association was found with the sleep hygiene score.

\section{Discussion}

\section{Findings}

In line with our hypothesis, CD patients used greater amount of thought suppression in comparison with the control group. As far as we are aware, no studies to date have directly investigated thought suppression in $\mathrm{CD}$ which we can directly compare our results with. However, previous reports suggested a "elective retrieval" of memories ${ }^{20}$ which was then associated with thought suppression evaluated by WBSI ${ }^{21}$. Furthermore, an fMRI study demonstrated neural correlates of a similar cognitive processing in $\mathrm{CD}^{8}$. These results are both in line with our findings and the classical "repression" of stressful memories and "conversion" into physical symptoms model. The more recent "integrative cognitive model" of $\mathrm{CD}$, having origins from the previous models, briefly proposes that symptoms of CD arise due to the preconscious "mismatching" of the stimuli with the mental representations ${ }^{1}$. Bridging with the classical theories, thought suppression may have a role on this mismatching process through inhibition of the retrieval of the closest match. On the other hand, objective evidence was found for increased arousal prior to the onset of the symptoms of $\mathrm{CD}$, followed by a reduction after the emergence ${ }^{22}$. Alternatively, thought suppression

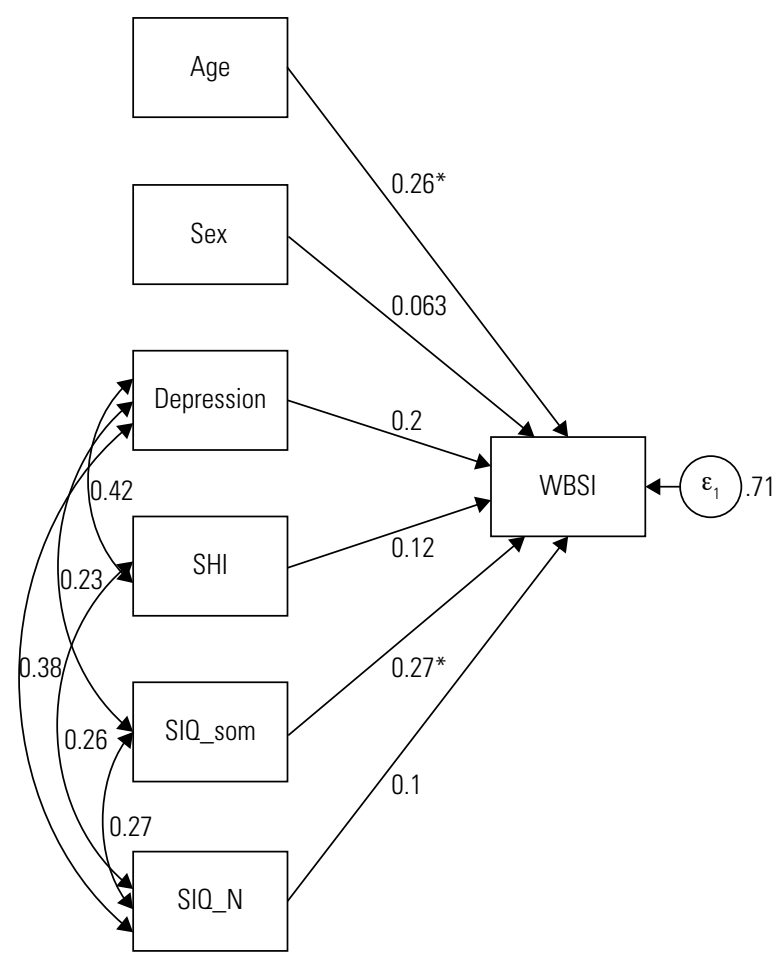

Figure 1. Structural equation model of the association of thought suppression with somatic attributions to the common bodily sensations, number of common bodily sensations, sleep hygiene, depressive symptoms, age and sex among conversion disorder patients. NOTE: Exogenously observed variables are inter-correlated in the model (15 covariance parameters exist), but only significant correlations are indicated in the diagram. Sex: 0 Male, 1 Female, Depression: Beck Depression Inventory Score, SHI: Sleep Hygiene Index Score, SIO som: Symptom Interpretation Questionnaire Somatic Attribution subscale score, SIO_ N: Symptom Interpretation Questionnaire number of symptoms; ${ }^{*} p<0.01$.

may be a compensation mechanism to the activation of this "rogue" representations and the related hyper-arousal state. Further studies are needed to shed light to these open questions.

In contrast to our hypothesis, patients with $\mathrm{CD}$ in isolation (without high depression scores) showed no significantly higher 
scores of thought suppression, suggesting the non-specificity of thought suppression to $\mathrm{CD}$. In line with this finding, thought suppression has been associated with various disorders including depression, anxiety disorders, obsessive compulsive disorder, PTSD and insomnia ${ }^{5,23,24}$. This result, taken together with the previous literature, suggests ubiquitous nature of the thought suppression.

In line with our hypothesis, thought suppression was significantly correlated with number of common bodily sensations and severity of depressive symptoms among CD patients, suggesting an association with the clinical severity of CD. Thought suppression was previously conceptualized as a representative of avoidance coping strategy usage, and associated with greater levels of rebound and higher levels of distress, thereof 5 . Relatedly, thought suppression may be considered as a non-specific marker of severity in CD. Prospective and/or experimental studies are needed to evaluate whether thought suppression has a causal role on the severity of the disorder or is only a factor that follows passively as a function of the general severity of psychopathology.

Our results replicated previous reports demonstrating poor sleep hygiene in $\mathrm{CD}^{10}$. However, incompatible with our hypothesis, we did not find a significant correlation between thought suppression and sleep hygiene scores among CD patients. Previous literature linked thought suppression with reduced quality of sleep 5 . Inconsistency with the previous studies may be explained by different samples, as none of these studies were conducted in CD samples. Interestingly, our results showed that sleep hygiene in $\mathrm{CD}$ was associated with psychological attributions rather than somatic or normalizing attributions, replicating a previous finding 25 . Hypothetically, sleep hygiene in CD may be associated with the content of the suppressed thought than the thought suppression process itself.

According to the SEM, older age and somatic attributions to the common bodily sensations were the significant correlates of thought suppression among CD patients. As far as we are aware, no studies to date have investigated the determinants of thought suppression in $\mathrm{CD}$ that enables a direct comparison. However, a tendency to focus on somatic causes of distress and suppress emotional components has been classically referred to $\mathrm{CD}^{1}$. In addition to this view, tendency to somatization seems to moderate the association between thought suppression and CD, and potentially the vice versa. Further researches with longitudinal designs are needed to test these associations.

CD patients show poorer performance in attention ${ }^{26}$. Relatedly, decline of attentional control by age was previously demonstrated ${ }^{27}$. Furthermore, poor attentional control and related mind-wandering was linked to thought suppression ${ }^{28}$. Through these results, the finding showing an increase of thought suppression by age in CD suggests the interpretation that thought suppression may be a compensation mechanism to mind-wandering related to alterations in attentional control by age. This interpretation is also in agreement with a recent study reporting increased externally-oriented thinking (which is related to thought suppression ${ }^{29}$ ) in older individuals compared to younger ${ }^{30}$. CD itself may be inducing the decline of attentional control in years, thereof facilitating the association between age and thought suppression. Studies longitudinally assessing the neuro-cognitive functioning and thought suppression in CD are needed to confirm these interpretations based on our preliminary results.

\section{Limitations}

First, due to the cross-sectional design of the study, causal inferences cannot be made. Second, our CD group consisted of individuals that applied to hospital. Therefore, these results cannot be generalized to the general population. Third, the CD and control group were matched for age, sex and neighborhood but unfortunately, not for educational level. However, educational level was used as a covariate in logistic regression models. Fourth, the analysis of thought suppression in comorbidity of $C D$ and depression was based on the high depression score variable obtained from the BDI. As it is not acceptable to make a diagnosis of clinical depression with a scale, the findings relying on this analysis should be considered as preliminary and needs to be confirmed in future research. Finally, the variables thought suppression, attributions to common bodily sensations and sleep hygiene are proxy variables and constructs, not variables per se. Therefore, a possible measurement error may occur. However, this is a limitation of all studies investigating these constructs. Furthermore, the interviews were carried out by clinicians who have remarkable experience on $\mathrm{CD}$ and the instruments used, minimizing the probability of measurement error.

\section{Conclusion}

The primary importance of this study is to be the first to explore thought suppression, symptom interpretation and sleep hygiene in CD. Briefly, the results revealed a non-specific association of thought suppression with CD. Furthermore, thought suppression was potentially associated with the clinical severity of CD. Finally, older age and somatic attributions to the common bodily sensations were the significant correlates of thought suppression among CD patients. Further research is needed to assess if thought suppression has a causal role on CD. These results take attention to psychosocial interventions that target thought suppression in CD.

\section{Funding}

This research received no specific grant from any funding agency in the public, commercial, or not-for-profit sectors.

\section{Disclosure}

All authors declare no conflict of interests.

\section{References}

1. Brown RJ, Reuber M. Towards an integrative theory of psychogenic non-epileptic seizures (PNES). Clin Psychol Rev. 2016;47:55-70.

2. Bowman ES. Why conversion seizures should be classified as a dissociative disorder. Psychiatr Clin North Am. 2006;29(1):185-211.

3. Nijenhuis ERS, Vanderlinden J, Spinhoven P. Animal defensive reactions as a model for trauma-induced dissociative reactions. J Traum Stress. 1998;11(2):243-60.

4. Carson AJ, Ringbauer B, MacKenzie L, Warlow C, Sharpe M. Neurological disease, emotional disorder, and disability: they are related: a study of 300 consecutive new referrals to a neurology outpatient department. J Neurol Neurosurg Psychiatry. 2000;68(2):202-6.

5. Najmi S, Wegner DM. Hidden complications of thought suppression. Int J Cogn Ther. 2009;2(3):210-23.

6. Kaplan MJ. A Psychodynamic Perspective on Treatment of Patients with Conversion and Other Somatoform Disorders. Psychodyn Psychiatry. 2014;42(4):593-615.

7. Ludwig L, Pasman JA, Nicholson T, Aybek S, David AS, Tuck S, et al. Stressful life events and maltreatment in conversion (functional neurological) disorder: systematic review and meta-analysis of case-control studies. Lancet Psychiatry. 2018;5(4):307-20.

8. Aybek S, Nicholson TR, Zelaya F, O'Daly OG, Craig TJ, David AS, et al. Neural correlates of recall of life events in conversion disorder. JAMA Psychiatry. 2014;71(1):52-60.

9. Brown RJ, Reuber M. Psychological and psychiatric aspects of psychogenic non-epileptic seizures (PNES): A systematic review. Clin Psychol Rev. 2016;45:157-82.

10. Graham CD, Kyle SD. A preliminary investigation of sleep quality in functional neurological disorders: Poor sleep appears common, and is associated with functional impairment. J Neurol Sci. 2017;378:163-6.

11. Hisli N. Use of the Beck Depression Inventory with Turkish University Students: Reliability, validity and Factor Analysis. Turk J Psychol. 1989;7:7-13.

12. Beck AT, Rush AJ, Shaw BF, Emery G. Cognitive therapy of depression. New York: Guilford Press; 1979.

13. Robbins JM, Kirmayer LJ. Attributions of common somatic symptoms. Psychol Med. 2009;21(4):1029-45.

14. Yenier Duman O, Usubütün S, Göka E. [Validity and reliability of the Turkish form of symptom interpretation questionnaire]. Turk Psikiyatri Derg. 2004;15(1):26-40. 
15. Wegner DM, Zanakos S. Chronic Thought Suppression. J Pers. 1994;62(4):615-40.

16. Ağargün MY, Beşiroğlu L, Kiran U, Kara H, Ozer O. The reliability and validity of the White Bear Suppression Inventory. Turk Psikiyatri Derg. 2004;15(4):282-90.

17. Mastin DF, Bryson J, Corwyn R. Assessment of Sleep Hygiene Using the Sleep Hygiene Index. J Behav Med. 2006;29(3):223-7.

18. Ozdemir PG, Boysan M, Selvi Y, Yildirim A, Yilmaz E. Psychometric properties of the Turkish version of the Sleep Hygiene Index in clinical and non-clinical samples. Compr Psychiatry. 2015;59:135-40.

19. Everitt B, Rabe-Hesketh S. Handbook of Statistical Analyses Using Stata. New York: Chapman and Hall/CRC; 2003.

20. Anderson MC. Neural Systems Underlying the Suppression of Unwanted Memories. Science. 2004;303(5655):232-5.

21. Pica G, Pierro A, Giannini A. The relationship between thought suppression and retrieval-induced forgetting: an analysis of witness memories. Cogn Process. 2014;16(1):35-44.

22. van der Kruijs SJM, Vonck KEJ, Langereis GR, Feijs LMG, Bodde NMG, Lazeron RHC, et al. Autonomic nervous system functioning associated with psychogenic nonepileptic seizures: Analysis of heart rate variability. Epilepsy Behav. 2016;54:14-9.
23. Ekinci O, Ekinci A. The relationship between clinical characteristics, metacognitive appraisals, and cognitive insight in patients with obsessive-compulsive disorder. Nord J Psychiatry. 2016;70(8):591-8.

24. Shipherd JC, Beck JG. The role of thought suppression in posttraumatic stress disorder. Behav Ther. 2005;36(3):277-87.

25. Storms MD, Nisbett RE. Insomnia and the attribution process. J Pers Soc Psychol. 1970;16(2):319-28.

26. Almis BH, Cumurcu BE, Unal S, Ozcan AC, Aytas O. The neuropsychological and neurophysiological profile of women with pseudoseizure. Compr Psychiatry. 2013;54(6):649-57.

27. Luo L, Craik FIM. Aging and memory: a cognitive approach. Can Psychiatry. 2008;53(6):346-53.

28. Smallwood J, Schooler JW. The restless mind. Psychol Bull. 2006;132(6):946-58.

29. Thompson BL, Waltz J. Mindfulness and experiential avoidance as predictors of posttraumatic stress disorder avoidance symptom severity. J Anxiety Disord. 2010;24(4):409-15.

30. Fani N, Skumlien M, Sederevicius D, Fjell AM, Walhovd KB, Westerhausen R. Parallel but independent reduction of emotional awareness and corpus callosum connectivity in older age. PLoS One. 2018;13(12):e0209915. 